\title{
LESSONS LEARNT FROM THE MANAGEMENT OF MULTIPLE CONTACTS OF A CASE OF PERTUSSIS IN A DELIVERY SUITE
}

\author{
Jennifer Vella and Krishna Hort \\ Western Sydney Area Public Health Unit

\section{Pauline Michell} \\ Wentworth Area Health Service.
}

The recent epidemic of pertussis in NSW has included many cases among adults. ${ }^{1}$ Delays in the diagnosis and notification of pertussis can result in the exposure of large numbers of susceptible persons, particularly when the case is a health worker. This article describes a case of pertussis in a health care worker who, while infectious, had multiple contacts with patients and their families in a maternity unit; and the response of the local public health unit and the hospital infection-control staff. The lessons learnt and an estimate of the costs are also presented.

\section{METHOD}

The index case was notified to the Wentworth Area Public Health Unit on Christmas Eve 1997, two weeks after laboratory diagnosis. Follow-up revealed that the case was a registered nurse who worked as a midwife in the delivery suite at a large district hospital. Onset of illness was estimated to be four weeks prior to notification. The case had attended work full-time for the three-week period they were infectious.

The standard definition for contacts of cases of pertussis used by the Western Sydney Area Public Health Unit is: 'any person with 20 hours or more face-to-face work, social or household contact with the case during the infectious period'. ${ }^{2}$ However, due to the vulnerability of neonates and the risk of transmission of Bordetella pertussis from infected mother to child, it was decided - in consultation with the AIDS and Infectious Disease Branch of the NSW Health Department - to expand the definition of contacts in this situation to include any exposure.

Identification of the mothers and babies who had been exposed to the case posed two problems. First, the case had worked between 12 and 14 shifts while infectious; however, the maternity unit was unable to identify those mothers, babies and support persons who had had prolonged contact with the case during that time. Second, mothers and their support persons could have been exposed to the case while receiving antenatal care in the clinic room, during monitoring in labour or while assisted during delivery.
Consequently, it was decided that all mothers admitted to the delivery suite or seen in the clinic room during the case's three-week infectious period, together with their newborn babies and any partners or family members present during labour, would be defined as contacts. They were advised by both telephone and letter to attend the Emergency Department at the Nepean Hospital to obtain a 10-day course of erythromycin. Also, staff who had come in close contact with the case were identified; advised to take a course of erythromycin; and provided with an information sheet on the disease.

The Emergency Department at the Nepean Hospital was informed that an influx of parents and their babies was likely during the Christmas holiday period. Pertussis information sheets, prescribing information and the limited available stock of erythromycin from the hospital pharmacy were sent to the Emergency Department.

\section{RESULTS}

A total of 149 mothers were contacted. Sixty-five mothers, 42 babies, 18 fathers, one grandmother and four siblings, were provided with erythromycin by the Emergency Department. Contacts who were on holiday were advised to obtain erythromycin from their local general practitioner or the nearest hospital. All were recommended to seek prompt medical attention if the symptoms of pertussis developed, and local medical practitioners were alerted to the situation. No secondary cases of pertussis were reported to the Western Sydney Area Public Health Unit in either mothers or babies in the following four weeks.

\section{COSTS INCURRED}

The cost of the intervention to the Western Sydney Area Health Service was estimated at $\$ 6000$, comprising: erythromycin provided to 151 contacts $(\$ 788.10)$; overtime for infection control and public health staff (3 days $=\$ 2000)$; telephone and postage $(\$ 250)$; and emergency staff time for providing erythromycin (100 person-hours $=\$ 3000)$.

\section{LESSONS LEARNT}

The following problems were encountered when tracing the contacts of the health care worker: 
- lack of staff to assist in contacting the exposed mothers by phone over the four-day Christmas holiday period;

- inadequate, incorrect, or out-of-date contact information for many mothers;

- overload of the Emergency Department with anxious parents during a busy holiday period;

- exhaustion of the hospital's stock of erythromycin and difficulty obtaining extra stock over the holiday period.

To avoid such problems in future, the Western Sydney Area Public Health Unit has developed a protocol for dealing with outbreaks of infectious disease. This protocol emphasises early recognition of the potential for large numbers of contacts, and identification of alternative options for implementing public health interventions, such as providing special facilities within the outpatients department or at community health centres.

\section{REFERENCES}

1. NSW Health Department. NSW Public Health Bulletin 1997; 8:8-9 (August-September, 1997), 73.

2. AIDS/Infectious Diseases Branch, NSW Health Department. Infectious Diseases Manual, 5th edn, Sydney: NSW Health Department, 1995.

\section{PERTUSSIS: A COMMENT}

\section{Peter B. McIntyre}

\section{Deputy Director}

National Centre for Immunisation Research and Surveillance of Vaccine Preventable Diseases New Children's Hospital, Westmead

The article by Vella et al. highlights the practical difficulties of managing exposure to pertussis in the health care setting. It is likely that other instances of exposure of newborn infants to pertussis in the health care setting have occurred in New South Wales during the period of highest pertussis activity in 1996-97. The method of diagnosis of the index case is not stated-a positive culture would have been of greater concern than positive serology alone. No data were presented describing compliance with the recommended course of erythromycin. Few of those who obtained erythromycin are likely to have completed a full 10-day course for themselves or their infants. However, erythromycin prophylaxis has been shown to be effective in the more intense exposure situation of mother to infant, ${ }^{1}$ and appears to have been effective in the present hospital outbreak. As pointed out by the authors, this incident prompted a public health response and raises at least two questions. Is the transmission of pertussis to patients (including but not limited to neonates) by health care workers a significant problem? What role is there for pertussis boosters for health care workers or adults in general?

The first report to draw attention to the then barely recognised problem of adult pertussis was from Sydney in $1978 .^{2}$ Australia currently has one of the highest notification rates for pertussis in persons over 20 years of age among countries with established pertussis immunisation programs. ${ }^{3}$ Adults are estimated to have been responsible for introducing infection into the household in approximately 15 per cent of cases. ${ }^{4}$ Although whole cell pertussis vaccine has been used successfully to terminate outbreaks in an institutional setting, it has generally been considered unsuitable for use in adults. ${ }^{5}$ Current infant/child acellular pertussis vaccines must also be modified for use in adults by reducing the amount of pertussis antigens as well as the diphtheria and tetanus content.

A trial of such a vaccine in 550 adults was recently carried out by the Centre for Immunisation Research at the New 